\title{
STABLE PROCESSES AND INTEGRAL EQUATIONS $\left({ }^{1}\right)$
}

\author{
BY \\ HAROLD WIDOM
}

\section{Introduction.}

1.1. Let $x(t), t \geqq 0$, be the symmetric stable process with exponent $\alpha$ $(0<\alpha \leqq 2)$, normalized so that the paths are continuous on the right, and starting at zero;

$$
E\left\{e^{i \xi x(t)}\right\}=e^{-t|\xi|^{\alpha}} .
$$

For a point $x_{0}$ with $-1<x_{0}<1$ set

$$
q\left(x_{0}, x, t\right)=\frac{d}{d x} \operatorname{Pr}\left\{\max _{\tau \leqq t}\left|x_{0}+x(\tau)\right|<1, x_{0}+x(t) \leqq x\right\} .
$$

In the case $\alpha=2$ (the Wiener process with variance 2) it is well known that

$$
q\left(x_{0}, x, t\right)=\sum_{j=1}^{\infty} e^{-\pi^{2} j^{2} t / 4} \phi_{j}\left(x_{0}\right) \phi_{j}(x)
$$

where

$$
\begin{aligned}
\phi_{2 k-1}(x) & =\pi^{-1 / 2} \cos (k-1 / 2) \pi x, \\
\phi_{2 k}(x) & =\pi^{-1 / 2} \sin k \pi x .
\end{aligned}
$$

In the case $\alpha=1$ it was shown by Kac and Pollard [7] that

$$
q\left(x_{0}, x, t\right)=\sum_{j=1}^{\infty} e^{-t / \lambda_{j}} \phi_{j}\left(x_{0}\right) \phi_{j}(x)
$$

where $\lambda_{j}$ and $\phi_{j}$ are the eigenvalues and normalized eigenfunctions of the integral equation on $(-1,1)$ with kernel

$$
\frac{1}{2 \pi} \log \frac{1-x y+\left[\left(1-x^{2}\right)\left(1-y^{2}\right)\right]^{1 / 2}}{1-x y-\left[\left(1-x^{2}\right)\left(1-y^{2}\right)\right]^{1 / 2}} .
$$

It is the main result of part II that (3) holds for any $\alpha$ in $0<\alpha<\leqq 2$, where now $\lambda_{j}$ and $\phi_{j}$ are the eigenvalues and normalized eigenfunctions of the integral equation on $(-1,1)$ with the symmetric kernel

Presented to the Society, August 29, 1960; received by the editors August 12, 1960.

(1) Supported by the National Science Foundation. 


$$
\begin{aligned}
K(x, y)= & \frac{\sec \alpha \pi / 2}{2 \Gamma(\alpha)}|x-y|^{\alpha-1} \\
& -\frac{\tan \alpha \pi / 2}{2 \pi \Gamma(\alpha)}\left(1-y^{2}\right)^{\alpha / 2} \int_{-1}^{1} \frac{(1-x t)^{\alpha-1}}{\left(1-t^{2}\right)^{\alpha / 2}(1-y t)} d t .
\end{aligned}
$$

Note that $K$ is not defined for $\alpha=1,2$, and in $\$ \$ 2.1$ and 2.2 we assume that $0<\alpha<1$ or $1<\alpha<2$. However, in $\$ 2.3$ we shall see that (3) remains valid when $K$ is defined at $\alpha=1$ (resp. 2) as its limit as $\alpha \rightarrow 1$ (resp. 2).

The symmetry of $K$, which is by no means obvious, will be established below. For $\alpha>1, K$ is continuous throughout the square; for $\alpha<1, K$ is continuous except on the diagonal $x=y$, and the reader can easily verify the estimate

$$
|K(x, y)| \leqq A|x-y|^{\alpha-1}
$$

where $A$ is a constant depending on $\alpha$. Thus in any case $K$ is the kernel of a self-adjoint completely continuous operator on $L_{2}(-1,1)$ so that the eigenfunctions $\phi_{j}$ form a complete set.

Since our contribution to the proof of (3) will be basically nothing more than a verification that $K$ satisfies the identity (14) below, we should indicate how $K$ was found. Denote the Laplace transform of $q$ by $Q$,

$$
Q\left(x_{0}, x, s\right)=\int_{0}^{\infty} e^{-s t} q\left(x_{0}, x, t\right) d t .
$$

It was shown by $\mathrm{Kac}[5]$ that in case $\alpha<1$ we have

$$
\int_{-1}^{1} Q\left(x_{0}, x, s\right)\left\{s \phi(x)+C(\alpha) \int_{-1}^{1} \frac{\phi^{\prime}(y) \operatorname{sgn}(x-y)}{|x-y|^{\alpha}} d y\right\} d x=\phi\left(x_{0}\right)
$$

for any $\phi$ satisfying $\phi( \pm 1)=0$ and $\phi^{\prime}(x) \in L_{2}(-1,1) ; C(\alpha)$ is a constant depending on $\alpha$. Now if we could find a complete orthonormal set of functions $\phi_{j}$ each of which satisfied $\phi_{j}( \pm 1)=0$ and satisfied

$$
\lambda_{j} \int_{-1}^{1} \frac{\phi_{j}^{\prime}(y) \operatorname{sgn}(x-y)}{|x-y|^{\alpha}} d y=\phi_{j}(x), \quad-1<x<1,
$$

then (7) would give immediately the Fourier coefficients of $Q$ relative to $\left\{\phi_{j}\right\}$. The problem is therefore the reduction of (8) to an ordinary integral equation. However the transform

$$
\int_{-1}^{1} \frac{\phi^{\prime}(y) \operatorname{sgn}(x-y)}{|x-y|^{\alpha}} d y=\Phi(x), \quad-1<x<1,
$$

can be inverted by a method of Carleman [1] to give $\phi^{\prime}$ in terms of $\Phi$. This inversion involves one arbitrary constant and the passage from $\phi^{\prime}$ to $\phi$ 
another-just enough to allow us to impose the conditions $\phi( \pm 1)=0$. With constant factors suitably adjusted the inversion formula turns out to be

$$
\phi(x)=\int_{-1}^{1} K\left(x, y^{\prime}\right) \Phi(y) d y
$$

with $K$ as given by (5). Although this method does (with a little more work) yield a proof of (3), we shall not use it. The main reason for this is that the method fails for $\alpha>1$, while a direct approach, knowing what the answer ought to be, takes care of all cases at once.

1.2. Let $T=T_{x_{0}}$ denote the first passage time of the process $x_{0}+x(t)$ to the exterior of the open unit interval;

$$
T=\min \left\{t:\left|x_{0}+x(t)\right| \geqq 1\right\} .
$$

The distribution function of $T$ can be expressed in terms of $q$. In fact

$$
\operatorname{Pr}\{T \leqq t\}=1-\int_{-1}^{1} q\left(x_{0}, x, t\right) d x .
$$

Although we do not know this explicitly, we can find all the moments of $T$ :

$$
\begin{aligned}
E\left\{T^{n}\right\} & =\int_{0}^{\infty} t^{n} d \operatorname{Pr}\{T \leqq t\} \\
& =n ! \sum \lambda_{j}^{n} \phi_{j}\left(x_{0}\right) \int_{-1}^{1} \phi_{j}(x) d x=n ! \int_{-1}^{1} K_{n}\left(x_{0}, x\right) d x
\end{aligned}
$$

where $K_{n}$ denotes the $n$th iterate of $K$. The first moment was obtained by Elliott [2] in case $0<\alpha<1$. Getoor [4] showed that Elliott's result holds for $1 \leqq \alpha \leqq 2$, and he found also the second moment. In principle his method could be used to find them all.

We can also express in terms of $q$ the joint distribution of $T$ and the place of first passage $x_{0}+x(T)$. It will be shown in $\$ 2.4$ that for $\alpha<2$ and $|y|>1$

$$
\begin{aligned}
\frac{d}{d y} \operatorname{Pr}\left\{x_{0}+x(T) \leqq y, T \leqq t\right\} & \\
=\frac{\sin \alpha \pi / 2}{\pi} & \left\{\frac{\left(1-x_{0}^{2}\right)^{\alpha / 2}}{\left(y^{2}-1\right)^{\alpha / 2}\left|y-x_{0}\right|}\right. \\
& \left.\quad-\left(y^{2}-1\right)^{-\alpha / 2} \int_{-1}^{1} \frac{\left(1-x^{2}\right)^{\alpha / 2}}{|y-x|} q\left(x_{0}, x, t\right) d x\right\} .
\end{aligned}
$$

In particular 


$$
\frac{d}{d y} \operatorname{Pr}\left\{x_{0}+x(T) \leqq y\right\}=\frac{1}{\pi} \sin \alpha \pi / 2 \frac{\left(1-x_{0}^{2}\right)^{\alpha / 2}}{\left(y^{2}-1\right)^{\alpha / 2}\left|y-x_{0}\right|} .
$$

This was obtained by Spitzer [11] in the case $\alpha=1$. The analogue of (10) for the one-sided absorption problem was obtained by Ray [10]. From (10) it is easy to compute the solution of the "ruin" problem, the probability of reaching (surpassing, if $\alpha<2$ ) 1 before -1 :

$$
\operatorname{Pr}\left\{x_{0}+x(T) \geqq 1\right\}=\frac{1}{2}+\frac{\Gamma\left(\frac{1+\alpha}{2}\right)}{\Gamma\left(\frac{1}{2}\right) \Gamma\left(\frac{\alpha}{2}\right)} x_{0} F\left(\frac{1}{2}, 1-\frac{\alpha}{2} ; \frac{3}{2} ; x_{0}^{2}\right),
$$

where $F$ denotes the usual hypergeometric function.

Most of the results just described have also been obtained by Getoor and H. Kesten, in papers to appear. In fact Getoor derived the distribution (10) of the place of first passage, and Kesten used this to obtain what we call $K(x, y)$. Thus the same theorems were proved, but in the order opposite to ours.

1.3. Let $X_{1}, X_{2}, \cdots$ be independent real valued random variables with the same symmetric density function $k(x)$ with finite variance $\sigma^{2}$. Set $S_{n}$ $=X_{1}+\cdots+X_{n}$. Then if $\left|x_{0}\right|<1$,

$$
\begin{aligned}
& \operatorname{Pr}\left\{\max _{k \leq^{n}}\left|x_{0}+n^{-1 / 2} S_{k}\right|<1\right\} \\
& =\int \cdots \int_{n^{1 / 2}\left(-1-x_{0}\right)}^{n^{1 / 8}\left(1-x_{0}\right)} k\left(x_{1}\right) k\left(x_{2}-x_{1}\right) \cdots k\left(x_{n}-x_{n-1}\right) d x_{1} \cdots d x_{n} \\
& =n^{n / 2} \int_{-1}^{1} \cdots \int_{-1}^{1} k\left(n^{1 / 2}\left(x_{1}-x_{0}\right)\right) \cdots k\left(n^{1 / 2}\left(x_{n}-x_{n-1}\right)\right) d x_{1} \cdots d x_{n} \\
& =\sum \mu_{j, n^{1 / 2}}^{n} \psi_{j, n^{1 / 2}}\left(x_{0}\right) \int_{-1}^{1} \psi_{j, n^{1 / 2}}(x) d x
\end{aligned}
$$

where we have denoted by $\mu_{j, A}$ and $\psi_{j, A}$ the eigenvalues and normalized eigenfunctions of the integral equation on $(-1,1)$ with kernel $A k(A(x-y))$. Now it is known [3] that

$$
\lim _{n \rightarrow \infty} \operatorname{Pr}\left\{\max _{t \leqq n}\left|x_{0}+n^{1 / 2} S_{k}\right|<1\right\}=\operatorname{Pr}\left\{\max _{t \leqq 1}\left|x_{0}+x(t)\right|<1\right\},
$$

where $x(t)$ is the Wiener process with variance $\sigma^{2}$. Thus

$$
\lim _{n \rightarrow \infty} \sum_{\mu_{j, n^{1 / 2}}^{n} \psi_{j, n^{1 / 2}}\left(x_{0}\right)} \int_{-1}^{1} \psi_{j, n^{1 / 2}}(x) d x=\sum_{j=1}^{\infty} e^{-\pi^{2} \sigma^{2} j^{2} / 8} \phi_{j}\left(x_{0}\right) \int_{-1}^{1} \phi_{j}(x) d x,
$$


where the $\phi_{j}$ are given by (2). This led to the conjecture $[6,81.7]$ that if $\mu_{1, A} \geqq \mu_{2, \Lambda} \geqq \cdots$ are the positive eigenvalues of $A k(A(x-y))$ then for each $j=1,2, \cdots$

or equivalently

$$
\lim _{A \rightarrow \infty} \frac{A_{j, A}^{2}}{\mu_{j, A}}=e^{-\pi^{20} \cos ^{2} / 8},
$$

$$
\mu_{j, A}=1-\frac{\pi^{2} \sigma^{2} j^{2}}{8 A^{2}}+o\left(A^{-2}\right) .
$$

This we proved in [12], with somewhat different assumptions on $k$ and by an entirely different method.

If $k$ does not have a finite second moment the method of [12] gives us no information whatever, although in certain cases the probabilistic argument given above yields a perfectly reasonable conjecture. Assume for example that

$$
\lim _{\xi \rightarrow 0} \frac{1-\hat{k}(\xi)}{|\xi|}=1
$$

where

$$
\hat{k}(\xi)=\int_{-\infty}^{\infty} e^{i \xi x} k(x) d x .
$$

Then in view of the theorem of Kac and Pollard [7] it was conjectured [6, 81.7] that in this case

$$
\mu_{j, A}=1-\lambda_{j}^{-1} A^{-1}+o\left(A^{-1}\right)
$$

where $\lambda_{1} \geqq \lambda_{2} \geqq \cdots$ are the eigenvalues of (4).

We shall show in part III of the present paper that the probabilistic argument can be pushed through. Thus using the result of part II we shall be able to determine the behavior of the $\mu_{j, A}$ whenever $1-k(\xi)$ behaves like $|\xi| \alpha(0<\alpha \leqq 2)$ near $\xi=0$. In fact, if

$$
\lim _{\xi \rightarrow 0} \frac{1-\hat{k}(\xi)}{|\xi|^{\alpha}}=c, \quad(0<c<\infty)
$$

then for each $j$

$$
\mu_{j, A}=1-c \lambda_{j}^{-1} A^{-\alpha}+o\left(A^{-\alpha}\right)
$$

as $A \rightarrow \infty$, where $\lambda_{j}$ is the $j$ th eigenvalue of the kernel $K(x, y)$ given by (5). Moreover the eigenfunctions $\psi_{j, A}$ of $A k(A(x-y))$ will approximate in some sense the eigenfunctions $\phi_{j}$ of $K$. The exact statement is given in $\S 3.1$. 
The probabilistic proof of these results has the disadvantage of not being extendable to the case when (11) holds with an $\alpha>2$. (Of course we no longer assume $k \geqq 0$.) We can conjecture what happens in this case, however, and do so in $\$ 3.5$.

Added in proof: The conjecture is proved in a forth coming paper.

II. Stable processes.

2.1. Let us define, for $|y|<1$ and all real $x$,

$$
\begin{aligned}
K_{0}(x, y)= & \frac{\sec \alpha \pi / 2}{2 \Gamma(\alpha)}|x-y|^{\alpha-1} \\
& -\frac{\tan \alpha \pi / 2}{2 \pi \Gamma(\alpha)}\left(1-y^{2}\right)^{\alpha / 2} \int_{|t|>1} \frac{|t-x|^{\alpha-1}}{\left(t^{2}-1\right)^{\alpha / 2}|t-y|} d t .
\end{aligned}
$$

We claim

$$
K_{0}(x, y)=\left\{\begin{array}{cc}
K(x, y), & |x| \leqq 1 \\
0, & |x| \geqq 1
\end{array}\right.
$$

The first identity is trivial, involving only a change of variable in the integral. The second is proved as follows. The function

$$
\frac{(t-x)^{\alpha-1}}{\left(t^{2}-1\right)^{\alpha / 2}}
$$

is analytic in $g t>0$, all relevant arguments being taken to lie between 0 and $\pi$. If we extend the function to the real axis by continuity, a simple contour integration shows

$$
\int_{-\infty}^{\infty} \frac{(t-x)^{\alpha-1}}{\left(t^{2}-1\right)^{\alpha / 2}(t-y)} d t=\pi i(y-x)^{\alpha-1}\left(y^{2}-1\right)^{-\alpha / 2},
$$

the integral being interpreted as a principal value. Assume $x \geqq 1$. Then (13) may be written

$$
\begin{aligned}
\left\{-\int_{-\infty}^{-1}-e^{i \pi \alpha / 2} \int_{-1}^{1}-e^{i \pi \alpha} \int_{1}^{x}+\int_{x}^{\infty}\right\} \frac{|t-x|^{\alpha-1}}{\left|t^{2}-1\right|^{\alpha / 2}(t-y)} d t \\
=-\pi i e^{i \pi \alpha / 2}(x-y)^{\alpha-1}\left(1-y^{2}\right)^{-\alpha / 2}
\end{aligned}
$$

If we multiply both sides by $e^{-i \pi \alpha / 2}$ and take imaginary parts, we obtain

$$
\sin \alpha \pi / 2 \int_{|t|>1} \frac{|t-x|^{\alpha-1}}{\left(t^{2}-1\right)^{\alpha / 2}|t-y|} d t=\pi(x-y)^{\alpha-1}\left(1-y^{2}\right)^{-\alpha / 2} .
$$

This shows $K_{0}(x, y)=0$ for $x \geqq 1$. Since $K_{0}(x, y)=K_{0}(-x,-y)$ we also have $K_{0}(x, y)=0$ for $x \leqq-1$.

The crucial identity is 


$$
\begin{aligned}
|\xi|^{\alpha} \int_{-1}^{1} K(x, y) e^{i \xi x} d x & =e^{i \xi y} \\
& -\frac{1}{\pi}(\sin \alpha \pi / 2)\left(1-y^{2}\right)^{\alpha / 2} \int_{|t|>1} \frac{e^{i \xi t}}{\left(t^{2}-1\right)^{\alpha / 2}|t-y|} d t .
\end{aligned}
$$

It will be seen in $\$ 2.2$ that for the proof of (3) the exact form of the second term on the right side of (14) is not important. What is important is that this term is the Fourier transform of a function vanishing on $(-1,1)$. In $\$ 2.4$, however, we shall need the full identity (14) for the computation of the distribution function of the place of first passage. To prove (14), assume first that $\alpha<1$. We have

$$
\int_{-\infty}^{\infty} e^{i \xi x}|x-y|^{\alpha-1} d x=2 \Gamma(\alpha)(\cos \alpha \pi / 2)|\xi|^{-\alpha} e^{i \xi \nu}
$$

Also,

$$
\begin{aligned}
\int_{-X}^{x} e^{i \xi x} d x & \int_{|t|>1} \frac{|t-x|^{\alpha-1}}{\left(t^{2}-1\right)^{\alpha / 2}|t-y|} d t \\
& =\int_{|t|>1} \frac{e^{i \xi t}}{\left(t^{2}-1\right)^{\alpha / 2}|t-y|} d t \int_{-x-t}^{x-t} e^{i \xi x}|x|^{\alpha-1} d x .
\end{aligned}
$$

As $X \rightarrow \infty$ the inner integral tends boundedly to $2 \Gamma(\alpha)(\cos \alpha \pi / 2)|\xi|^{-\alpha}$. Consequently

$$
\begin{aligned}
& \int_{-\infty}^{\infty} e^{i \xi x} d x \int_{|t|>1} \frac{|t-x|^{\alpha-1}}{\left(t^{2}-1\right)^{\alpha / 2}|t-y|} d t \\
& \quad=2 \Gamma(\alpha)(\cos 2 \pi / 2)|\xi|^{-\alpha} \int_{|t|>1} \frac{e^{i \xi t}}{\left(t^{2}-1\right)^{\alpha / 2}|t-y|} d t .
\end{aligned}
$$

The identities (12), (15), and (16) give (14). That (14) holds also for $\alpha>1$ follows from the fact that for fixed $\xi$ and $y$ both sides are analytic in $0<R \alpha<2$ (except possibly for $\alpha=1$ ).

We now show that $K$, the integral operator on $L_{2}(-1,1)$ with kernel $K(x, y)$, is positive definite. Assume that $K f=0$. If we multiply both sides of (14) by $f(y)$ and integrate with respect to $y$ we obtain

$$
0=\hat{f}(\xi)-\hat{g}(\xi),
$$

where $g(x)$ vanishes on $(-1,1)$ and is the $L_{1}$ function

$$
\frac{1}{\pi}(\sin \alpha \pi / 2)\left(x^{2}-1\right)^{-\alpha / 2} \int_{-1}^{1}\left(1-y^{2}\right)^{\alpha / 2} \frac{f(y)}{|x-y|} d y
$$


outside $(-1,1)$. Consequently $f=0$. Next assume $\alpha<1$ and let $f$ be any function in $L_{2}(-1,1)$ vanishing near \pm 1 . We obtain from (14)

$$
|\xi|^{\alpha}(K f)-(\xi)=\hat{f}(\xi)-\hat{g}(\xi)
$$

and $g \in L_{2}$. Therefore

$$
(K f, f)=(K f, f-g)=\frac{1}{2 \pi}((K f) \widehat{\jmath}, \hat{f}-\hat{g})=\frac{1}{2 \pi}\left((K f)^{\curlywedge},|\xi| \alpha(K f) \uparrow\right) \geqq 0 .
$$

The argument must be modified if $\alpha>1$ since $g$ does not necessarily belong to $L_{2}$. However in this case the kernel is bounded so that $K f \in L_{\infty}$. Introduce the functions

$$
h_{\sigma}(x)=(\pi \sigma)^{-1 / 2} e^{-x^{2} / \sigma}, \quad \hat{h}_{\sigma}(\xi)=e^{-\sigma \xi^{2} / 4} .
$$

Then we have (the asterisk denotes convolution)

$$
\begin{aligned}
(\boldsymbol{K} f, f) & =(\boldsymbol{K} f, f-g)=\lim _{\sigma \rightarrow 0}\left(\boldsymbol{K} f,(f-g) * h_{\sigma}\right) \\
& =\frac{1}{2 \pi} \lim _{\sigma \rightarrow 0}\left((\boldsymbol{K} f)^{-},(\hat{f}-\hat{g}) \hat{h}_{\sigma}\right)=\frac{1}{2 \pi} \lim _{\sigma \rightarrow 0}\left((\boldsymbol{K} f)^{-},|\xi|^{\alpha}(\boldsymbol{K} f)^{-} \hat{h}_{\sigma}\right) \geqq 0
\end{aligned}
$$

since $\hat{h}_{\sigma}>0$. Thus in any case $K$ is (symmetric and) positive definite.

2.2. We can now prove (3). We use the following fact: If $g$ is a continuous function on $(-1,1)$ and

$$
|\xi| \alpha \hat{g}(\xi)=\hat{h}(\xi),
$$

where $h$ is an $L_{1}$ function bounded near $x=x_{0}$, then

$$
\int_{-1}^{1} Q\left(x_{0}, x, s\right)[s g(x)+h(x)] d x=g\left(x_{0}\right) \text {. }
$$

(See [5], where a variant of this is derived.) Now take for $g$ an eigenfunction $\phi_{j}$ of $K$. (The eigenfunctions are continuous since a sufficiently high iterate of $K$ is continuous on the square.) Then from (14)

$$
\begin{aligned}
\lambda_{j}|\xi|^{\alpha} \hat{\phi}_{j}(\xi)= & \hat{\phi}_{j}(\xi) \\
& -\frac{1}{\pi} \sin \alpha \pi / 2 \int_{|t|>1} \frac{e^{i \xi t}}{\left(t^{2}-1\right)^{\alpha / 2}} d t \int_{-1}^{1} \frac{\left(1-y^{2}\right)^{\alpha / 2}}{|t-y|} \phi_{j}(y) d y,
\end{aligned}
$$

so we have (17) with $h(x)$ equal to $\lambda_{j}^{-1} \phi_{j}(x)$ on $|x|<1$ and to

$$
-\left(\pi \lambda_{j}\right)^{-1}(\sin \alpha \pi / 2)\left(x^{2}-1\right)^{-\alpha / 2} \int_{-1}^{1} \frac{\left(1-y^{2}\right)^{\alpha / 2}}{|x-y|} \phi_{j}(y) d y
$$

on $|x|>1$; moreover $h \in L_{1}$ and is bounded except possibly near \pm 1 . We conclude from (18) 


$$
\left(s+\lambda_{j}^{-1}\right) \int_{-1}^{1} Q\left(x_{0}, x, s\right) \phi_{j}(x) d x=\phi_{j}\left(x_{0}\right)
$$

and so

$$
q\left(x_{0}, x, t\right)=\sum_{j=1}^{\infty} e^{-t / \lambda_{j}} \phi_{j}\left(x_{0}\right) \phi_{j}(x) .
$$

Note that since some iterate of $K$ is continuous, Mercer's theorem implies that the series in (3) converges absolutely and uniformly in $-1<x, x_{0}<1$ for each $t>0$.

2.3. A few words are in order concerning the cases $\alpha=1,2$. From (13) one easily derives the identity

$$
\begin{aligned}
K(x, y)= & \frac{\cos \alpha \pi / 2}{\Gamma(\alpha)}|x-y|^{\alpha-1} \max (0, x-y) \\
& -\frac{\sin \alpha \pi / 2}{\pi \Gamma(\alpha)}\left(1-y^{2}\right)^{\alpha / 2} \int_{-1}^{x} \frac{(x-t)^{\alpha-1}}{\left(1-t^{2}\right)^{\alpha / 2}(t-y)} d t
\end{aligned}
$$

Now although $K$ is not defined for $\alpha=1$ the right side of (20) is. To see that we have proved (3), with the right side of (20) as our kernel, even for $\alpha=1$, note that only two things are necessary: an estimate on the kernel (the inequality $|K(x, y)| \leqq A \log \left(1+|x-y|^{-1}\right)$ is easy for $x<y$ and follows for all $x, y$ from the symmetry of $K$ ) and the identity (14) (which follows by continuity from the identity for $\alpha \neq 1$ ). We did not use the right side of (20) as our kernel all along, and so avoid this special argument, because the original form of $K$ was more convenient for computation. The reader will have no difficulty verifying that for $\alpha=1$ the right side of (20) is equal to (4).

As for $\alpha=2$, it is not hard to see that

$$
\lim _{\alpha \rightarrow 2} K(x, y)=\frac{1}{2}[1+\min (x, y)][1-\max (x, y)] .
$$

Thus if $K$ is defined by the right side of (21) for $\alpha=2$ the identity (3) reduces to the known identity (1).

2.4. We shall now derive (9). If we set

$$
p\left(x_{0}, x, t\right)=\frac{d}{d x} \operatorname{Pr}\left\{x_{0}+x(t) \leqq x\right\}=\frac{1}{2 \pi} \int_{-\infty}^{\infty} e^{i \xi\left(x-x_{0}\right)} e^{-t|\xi|^{\alpha}} d \xi
$$

then we have (see [10])

$$
\begin{aligned}
p\left(x_{0}, x, t\right)= & q\left(x_{0}, x, t\right) \\
& +\iint_{0 \leq t^{\prime} \leq t,|y| \geq 1} p\left(y, x, t-t^{\prime}\right) d_{y, t^{\prime}} \operatorname{Pr}\left\{x_{0}+x(T) \leqq y, T \leqq t^{\prime}\right\} .
\end{aligned}
$$


If we multiply both sides by $e^{i \xi x} \phi_{j}\left(x_{0}\right)$ and integrate with respect to $x$ from $-\infty$ to $\infty$ and with respect to $x_{0}$ from -1 to 1 we obtain, using (3), $e^{-t|\xi|^{\alpha}} \hat{\phi}_{j}(\xi)=e^{-t / \lambda_{j}} \hat{\phi}_{j}(\xi)$

$+\iiint_{0 \leq t^{\prime} \leq t,|y| \geq 1,\left|x_{0}\right| \leq 1} e^{i \xi y} e^{-\left(t-t^{\prime}\right)|\xi|^{\alpha} \phi_{j}\left(x_{0}\right) d_{y, t^{\prime}}} \operatorname{Pr}\left\{x_{0}+x(T) \leqq y, T \leqq t^{\prime}\right\} d x_{0}$.

Now take the Laplace transform of both sides:

$$
\begin{aligned}
& \frac{\hat{\phi}_{j}(\xi)}{s+|\xi|^{\alpha}}=\frac{\hat{\phi}_{j}(\xi)}{s+\lambda_{j}^{-1}} \\
& +\frac{1}{s+|\xi|^{\alpha}} \iiint_{t \geq 0,|y| \geq 1,\left|x_{0}\right| \leq 1} e^{-s t} e^{i \xi v} \phi_{j}\left(x_{0}\right) d_{y, t} \operatorname{Pr}\left\{x_{0}+x(T) \leqq y, T \leqq t\right\} d x_{0} .
\end{aligned}
$$

Therefore, using (19),

$$
\begin{aligned}
\iint_{t \geq 0,|y| \geq 1} e^{-s t} e^{i \xi y} d_{t, y} \int_{-1}^{1} \operatorname{Pr}\left\{x_{0}+x(T) \leqq y, T \leqq t\right\}_{j}\left(x_{0}\right) d x_{0} \\
\quad=\frac{1-\lambda_{j}|\xi|^{\alpha}}{1+\lambda_{j} s} \hat{\phi}_{j}(\xi) \\
=\frac{\sin \alpha \pi / 2}{\pi\left(1+\lambda_{j} s\right)} \int_{|y| \geq 1} \frac{e^{i \xi y}}{\left(y^{2}-1\right)^{\alpha / 2}} d y \int_{-1}^{1} \frac{\left(1-x^{2}\right)^{\alpha / 2}}{|y-x|} \phi_{j}(x) d x \\
=-\frac{\sin \alpha \pi / 2}{\pi} \int_{|y| \geq 1} \frac{e^{i \xi y}}{\left(y^{2}-1\right)^{\alpha / 2}} d y \int_{0}^{\infty} e^{-s t} d_{t} e^{-t / \lambda_{j}} \int_{-1}^{1} \frac{\left(1-x^{2}\right)^{\alpha / 2}}{|y-x|} \phi_{j}(x) d x .
\end{aligned}
$$

It follows that

$$
\begin{aligned}
& \int_{-1}^{1} \operatorname{Pr}\left\{x_{0}+x(T) \leqq y, T \leqq t\right\} \phi_{j}\left(x_{0}\right) d x_{0} \\
& \quad=C-\frac{\sin \alpha \pi / 2}{\pi} e^{-t / \lambda_{j}} \int_{|\eta| \geq 1, \eta \leq \nu} \frac{d \eta}{\left(\eta^{2}-1\right)^{\alpha / 2}} \int_{-1}^{1} \frac{\left(1-x^{2}\right)^{\alpha / 2}}{|\eta-x|} \phi_{j}(x) d x
\end{aligned}
$$

where $C$ is independent of $t$. Now as $t \rightarrow 0, \operatorname{Pr}\left\{T=T_{x_{0}} \leqq t\right\} \rightarrow 0$ weakly on $-1<x_{0}<1$; for

$$
1-\operatorname{Pr}\{T \leqq t\}=\sum e^{-t / \lambda_{j}} \phi_{j}\left(x_{0}\right) \int_{-1}^{1} \phi_{j}(x) d x
$$

so

$$
\int_{-1}^{1}(1-\operatorname{Pr}\{T \leqq t\}) \phi_{j}\left(x_{0}\right) d x_{0}=e^{-t / \lambda_{j}} \int_{-1}^{1} \phi_{j}(x) d x \rightarrow \int_{-1}^{1} \phi_{j}\left(x_{0}\right) d x_{0}
$$


Since the $\phi_{j}$ are complete this shows $1-\operatorname{Pr}\{T \leqq t\} \rightarrow 1$ weakly. It follows that as $t \rightarrow 0$ the left side of (22) approaches zero. Hence

$$
\begin{aligned}
\int_{-1}^{1} \operatorname{Pr}\left\{x_{0}\right. & +x(T) \leqq y, T \leqq t\} \phi_{j}\left(x_{0}\right) d x_{0} \\
& =\frac{\sin \alpha \pi / 2}{\pi}\left(1-e^{-t / \lambda_{j}}\right) \int_{|\eta| \geq 1, \eta \leq \nu} \frac{d \eta}{\left(\eta^{2}-1\right)^{\alpha / 2}} \int_{-1}^{1} \frac{\left(1-x^{2}\right)^{\alpha / 2}}{|\eta-x|} \phi_{j}(x) d x \\
& =\frac{\sin \alpha \pi / 2}{\pi} \int_{|\eta| \geq 1, \eta \leq \nu} \frac{d \eta}{\left(\eta^{2}-1\right)^{\alpha / 2}} \int_{-1}^{1} \frac{\left(1-x^{2}\right)^{\alpha / 2}}{|\eta-x|}\left\{\phi_{j}(x)\right. \\
& \left.-\int_{-1}^{1} q\left(x_{0}, x, t\right) \phi_{j}\left(x_{0}\right) d x_{0}\right\} d x .
\end{aligned}
$$

Therefore

$$
\begin{aligned}
\operatorname{Pr}\left\{x_{0}+x(T) \leqq y, T \leqq t\right\} & \\
= & \frac{\sin \alpha \pi / 2}{\pi}\left\{\left(1-x_{0}^{2}\right)^{\alpha / 2} \int_{|\eta| \geq 1, \eta \leq \nu} \frac{d \eta}{\left(\eta^{2}-1\right)^{\alpha / 2}\left|\eta-x_{0}\right|}\right. \\
& \left.-\int_{|\eta| \geq 1, \eta \leq y} \frac{d \eta}{\left(\eta^{2}-1\right)^{\alpha / 2}} \int_{-1}^{1} \frac{\left(1-x^{2}\right)^{\alpha / 2}}{|\eta-x|} q\left(x_{0}, x, t\right) d x\right\},
\end{aligned}
$$

which gives (9).

III. Integral equations.

3.1. We consider a non-negative even function $k$ which satisfies the conditions

$$
\int_{-\infty}^{\infty} k(x) d x=1, \quad \lim _{\xi \rightarrow 0} \frac{1-k(\xi)}{|\xi|^{\alpha}}=c \quad(0<c<\infty)
$$

with $0<\alpha \leqq 2$. Denote by $\mu_{1, \Lambda} \geqq \mu_{2, \Lambda} \geqq \cdots$ and $\psi_{1, \Lambda}, \psi_{2, \Lambda}, \cdots$ the positive eigenvalues and corresponding normalized eigenfunctions of the integral equation

$$
A \int_{-1}^{1} k(A(x-y)) \psi(y) d y=\mu \psi(x), \quad-1<x<1 .
$$

Our main result here is that for fixed $j=1,2, \ldots$

$$
\mu_{j, A}=1-c \lambda_{j}^{-1} A^{-\alpha}+o\left(A^{-\alpha}\right)
$$

as $A \rightarrow \infty$,

where $\lambda_{j}$ (and $\phi_{j}$ ) are as before.

We would also like to say that $\psi_{j, A} \rightarrow \phi_{j}$ for each $j$. To prove this we need the simplicity of the $\lambda_{j}$, which unfortunately we have been unable to establish (except of course in case $\alpha=2$ when $\lambda_{j}=4 / \pi^{2} j^{2}$ ). We therefore content our- 
selves with the following substitute. Group the positive integers into blocks $J_{1}, J_{2}, \cdots$ so that indices in the same block correspond to equal $\lambda$ 's while indices in different blocks correspond to unequal $\lambda$ 's. Thus $\lambda_{j_{1}}=\lambda_{j_{2}}$ if and only if $j_{1}$ and $j_{2}$ belong to the same block $J$. Let $\mathfrak{T}_{i}$ be the space spanned by the $\phi_{j}$ with $j \in J_{i}$ and $\mathfrak{r}_{i, A}$ the space spanned by the $\psi_{j, A}$ with $j \in J_{i}$. We shall prove that for fixed $i$ the space $\mathfrak{N}_{i, A}$ converges in mean square to $\mathfrak{M}_{i}$ as $A \rightarrow \infty$. That is, given $\epsilon>0$ there is an $A_{0}$ such that

(i) For any $A \geqq A_{0}$ and $\phi \in \mathfrak{N}_{i}$ there is a $\psi \in \mathscr{N}_{i, A}$ with

$$
\int_{-1}^{1}|\phi(x)-\psi(x)|^{2} d x \leqq \epsilon^{2}
$$

(ii) For any $A \geqq A_{0}$ and $\psi \in \mathfrak{N}_{i, A}$ there is a $\phi \in \mathfrak{N}_{i}$ so that (23) holds.

Observe that if $\lambda_{j}$ is simple, and therefore for any $j$ if $\alpha=2$, we can conclude that the $\psi_{j, A}$ (multiplied by suitable constants of absolute value one) converge in mean square to $\phi_{j}$.

3.2. In what follows we assume $c=1$; passage to the general case follows by replacing $k(x)$ by $c^{-1 / \alpha} k\left(c^{-1 / \alpha} x\right)$. Let $X_{1}, X_{2}, \cdots$ be independent random variables having the same density function $k$, and set $S_{n}=X_{1}+\cdots+X_{n}$. We assert that if $m$ is a positive integer and $-1<x_{0}, y<1$ then

$$
\begin{array}{r}
\lim _{A \rightarrow \infty} \operatorname{Pr}\left\{\max _{k \leqq\left[A^{\alpha}\right] m}\left|x_{0}+A^{-1} S_{k}\right|<1, x_{0}+A^{-1} S_{\left[A^{\alpha}\right] m} \leqq y\right\} \\
=\operatorname{Pr}\left\{\max _{t \leqq m}\left|x_{0}+x(t)\right|<1, x_{0}+x(m) \leqq y\right\}
\end{array}
$$

where $x(t)$ is the symmetric stable process with exponent $\alpha$. Unfortunately we have been unable to find in the literature a statement which contains (24), but results of Kimme [8] come very close. In our case these results (Theorems 6 and 9 of [8]) imply the following:

Assume that as $n \rightarrow \infty, \nu(n) \sim n^{-1 / \alpha}$, and set

$$
x_{n}(t)=\nu(n) S_{[t n]}, \quad 0 \leqq t \leqq 1 .
$$

Let $F$ be a functional defined, bounded, and continuous in the uniform topology of the space of bounded functions on $[0,1]$. Assume further that $F$ has the following property $P$ : If $f_{n}$ are uniformly bounded and converge, except on a denumerable set, to $f$, then $F\left[f_{n}\right] \rightarrow F[f]$. The conclusion is

$$
\lim _{n \rightarrow \infty} E\left\{F\left[x_{n}\right]\right\}=E\{F[x]\} \text {. }
$$

Now we are of course interested in the functional $F_{0}$ defined by

$$
F_{0}[f]= \begin{cases}1, & \text { if } \sup _{0 \leqq t \leqq 1}\left|x_{0}+f(t)\right|<1 \text { and } x_{0}+f(1) \leqq y \\ 0, & \text { otherwise. }\end{cases}
$$


This functional is hopelessly discontinuous so we define continuous approximants. For $\epsilon>0$ set

$$
\begin{aligned}
& g_{\epsilon}(x)=\left\{\begin{array}{ll}
1, & x \leqq 1, \\
0, & x \geqq 1+\epsilon, \\
\text { linear, } & 1 \leqq x \leqq 1+\epsilon,
\end{array} g_{-\epsilon}(x)=\left\{\begin{array}{ll}
1, & x \leqq 1-\epsilon, \\
0, & x \geqq 1, \\
\text { linear, } & 1-\epsilon \leqq x \leqq 1,
\end{array} \quad h_{-\epsilon}(x)= \begin{cases}1, & x \leqq y, \\
0, & x \geqq y+\epsilon, \\
\text { linear, } & y \leqq x \leqq y+\epsilon,\end{cases} \right.\right. \\
& F_{ \pm \epsilon}[f]=g_{ \pm \epsilon}\left(\sup _{0 \leqq t \leqq 1}\left|x_{0}+f(t)\right|\right) h_{ \pm \epsilon}\left(x_{0}+f(1)\right) . \\
& \text { linear, }
\end{aligned}
$$

Then the functionals $F_{ \pm \epsilon}$ are bounded and continuous in the uniform topology of bounded functions on $[0,1]$, although they still do not have property $P$. Fortunately property $P$ is not necessary. Examination of the proof of Theorem 9 of [8] shows that $P$ may be replaced by $P^{\prime}$ :

For almost all sample functions $x(t)$ we have the following. Let

$$
\begin{aligned}
x_{N}^{*}(t) & =\sup _{(j-1) / N<t \leqq j / N} x(t), \\
x_{N}^{* *}(t) & =\inf _{(j-1) / N<t \leqq j / N} x(t),
\end{aligned}
$$

If for each $N$ we have $x_{N}^{* *} \leqq f_{N} \leqq x_{N}^{*}$, then $F\left[f_{N}\right] \rightarrow F[x]$.

Now our functionals $F_{ \pm \epsilon}$ have property $P^{\prime}$, so

$$
\lim _{n \rightarrow \infty} E\left\{F_{ \pm \epsilon}\left[x_{n}\right]\right\}=E\left\{F_{ \pm \epsilon}[x]\right\} \text {. }
$$

Consequently

$$
\begin{aligned}
E\left\{F_{-\epsilon}[x]\right\} & =\lim _{n \rightarrow \infty} E\left\{F_{-\epsilon}\left[x_{n}\right]\right\} \leqq \liminf _{n \rightarrow \infty} E\left\{F_{0}\left[x_{n}\right]\right\} \\
& \leqq \limsup _{n \rightarrow \infty} E\left\{F_{0}\left[x_{n}\right]\right\} \leqq \lim _{n \rightarrow \infty} E\left\{F_{\epsilon}\left[x_{n}\right]\right\}=E\left\{F_{\epsilon}[x]\right\} .
\end{aligned}
$$

As $\epsilon \rightarrow 0$ the extreme terms in the inequality both converge to $E\left\{F_{0}[x]\right\}$. Therefore

$$
\lim _{n \rightarrow \infty} E\left\{F_{0}\left[x_{n}\right]\right\}=E\left\{F_{0}[x]\right\} .
$$

Now using the definitions of $F_{0}$ and $x_{n}$, and observing that in Kimme's work the $t$-interval $[0,1]$ could have been replaced by $[0, m]$, we obtain 


$$
\begin{gathered}
\lim _{n \rightarrow \infty} \operatorname{Pr}\left\{\max _{k \leqq n m}\left|x_{0}+\nu(n) S_{k}\right|<1, x_{0}+\nu(n) S_{m n} \leqq y\right\} \\
=\operatorname{Pr}\left\{\max _{t \leqq m}\left|x_{0}+x(t)\right|<1, x_{0}+x(m) \leqq y\right\} .
\end{gathered}
$$

Take any sequence of $A^{\prime}$ 's going to infinity and set $n=\left[A^{\alpha}\right], \nu(n)=A^{-1}$. We obtain (24).

3.3. In $\S 3.1$ we denoted by $\mu_{j, A}$ and $\psi_{j, A}(j=1,2, \cdots)$ the positive eigenvalues and corresponding eigenfunctions of $A k(A(x-y))$. There may be other eigenvalues. We denote these, and the corresponding eigenfunctions, by $\mu_{j, A}$ and $\psi_{j, A}$ with $j=0,-1, \cdots$. Their ordering is irrelevant. We have

$$
\begin{aligned}
\operatorname{Pr}\left\{\max _{k \leqq\left[A^{\alpha}\right] m}\left|x_{0}+A^{-1} S_{k}\right|\right. & \left.<1, x_{0}+A^{-1} S_{\left[A^{\alpha}\right] m} \leqq y\right\} \\
& =\sum_{j=-\infty}^{\infty} \mu_{j, A}^{[A \alpha] m} \psi_{j, A}\left(x_{0}\right) \int_{-1}^{y} \psi_{j, A}(x) d x .
\end{aligned}
$$

Therefore (24) and (3) give

$$
\lim _{A \rightarrow \infty} \sum_{j=-\infty}^{\infty} \mu_{j, A}^{\left[A^{\alpha}\right] m} \psi_{j}\left(x_{0}\right) \int_{-1}^{y} \psi_{j}(x) d x=\sum_{j=1}^{\infty} e^{-m / \lambda_{j}} \phi_{j}\left(x_{0}\right) \int_{-1}^{y} \phi_{j}(x) d x .
$$

Since this holds for $m=1,2, \cdots$ we have

$$
\lim _{A \rightarrow \infty} \sum_{j=-\infty}^{\infty} P\left(\mu_{j, A}^{[A \alpha]}\right) \psi_{j, A}\left(x_{0}\right) \int_{-1}^{y} \psi_{j, A}(x) d x=\sum_{j=1}^{\infty} P\left(e^{-1 / \lambda_{j}}\right) \phi_{j}\left(x_{0}\right) \int_{-1}^{y} \phi_{j}(x) d x
$$

for any polynomial $P(u)$ vanishing at $u=0$. Now for any function $F(u)$ defined on $(-1,1)$ we have

$$
\begin{aligned}
& \int_{-1}^{1}\left|\sum_{j=-\infty}^{\infty} F\left(\mu_{j, A}^{\left[A^{\alpha}\right]}\right) \psi_{j, A}\left(x_{0}\right) \int_{-1}^{y} \psi_{j, A}(x) d x\right|^{2} d x_{0} \\
& =\sum_{j=-\infty}^{\infty} F\left(\mu_{j, A}^{\left[A^{\alpha}\right]}\right)^{2}\left\{\int_{-1}^{y} \psi_{j, A}(x) d x\right\}^{2} \leqq \max _{|u| \leqq 1}\left(\frac{F(u)}{u}\right)^{2} \sum_{j=-\infty}^{\infty} \mu_{j, A}^{2\left[A^{\alpha}\right]}\left\{\int_{-1}^{y} \psi_{j, A}(x) d x\right\}^{2} \\
& =\max _{|u| \leqq 1}\left(\frac{F(u)}{u}\right)^{2} \int_{-1}^{1}\left|\sum_{j=-\infty}^{\infty} \mu_{j, A}^{\left[A^{\alpha}\right]} \psi_{j, A}\left(x_{0}\right) \int_{-1}^{y} \psi_{j, A}(x) d x\right|^{2} d x_{0} \\
& =\max _{|u| \leqq 1}\left(\frac{F(u)}{u}\right)^{2} \int_{-1}^{1}\left(\operatorname{Pr}\left\{\max _{k \leqq\left[A^{\alpha}\right]}\left|x_{0}+A^{-1} S_{k}\right|<1, x_{0}+A^{-1} S_{\left[A^{\alpha}\right]} \leqq y\right\}\right)^{2} d x_{0} \\
& \leqq 2 \max _{|u| \leqq 1}\left(\frac{F(u)}{u}\right)^{2} .
\end{aligned}
$$

Similarly 


$$
\int_{-1}^{1}\left|\sum_{j=1}^{\infty} F\left(e^{-1 / \lambda_{j}}\right) \phi_{j}\left(x_{0}\right) \int_{-1}^{y} \phi_{j}(x) d x\right|^{2} d x_{0} \leqq 2 \max _{|u| \leqq 1}\left(\frac{F(u)}{u}\right)^{2} .
$$

In view of these inequalities, (25) holds for any function $P(u)$ which is continuous on $(-1,1)$ and such that $\lim _{u \rightarrow 0} P(u) / u$ exists. The two sums are interpreted as being mean square limits over $-1<x_{0}<1$ of the finite partial sums, and the limit as $A \rightarrow \infty$ is also interpreted as being in mean square.

3.4. To pass from (25) to the theorems stated in $\$ 3.1$ we prove a series of four lemmas.

LEMma 1. A ssume that for each of a sequence of $A$ 's tending to infinity $\mu_{A}$ is an eigenvalue and $\psi_{A}$ a corresponding normalized eigenfunction of $A k(A(x-y))$. Assume further that the $\mu_{A}$ satisfy an inequality

$$
\mu_{A} \geqq 1-\gamma A^{-\alpha} \quad(\gamma>0) .
$$

Then weak convergence of the $\psi_{A}$ implies mean square convergence.

Proof. Set

$$
\Psi_{A}(\xi)=(2 \pi)^{-1 / 2} \int_{-1}^{1} e^{i \xi x} \psi_{A}(x) d x
$$

Then Parseval's identity applied to the equation

$$
\mu_{A} \psi_{A}(x)=A \int_{-1}^{1} k(A(x-y)) \psi_{A}(y) d y, \quad-1<x<1,
$$

gives

$$
\mu_{A}=\mu_{A} \int_{-\infty}^{\infty}\left|\Psi_{A}(\xi)\right|^{2} d \xi=\int_{-\infty}^{\infty} \hat{k}\left(\frac{\xi}{A}\right)\left|\Psi_{A}(\xi)\right|^{2} d \xi .
$$

Pick a $\Delta>0$. Then for $A$ sufficiently large we shall have

$$
\hat{k}\left(\frac{\xi}{A}\right) \leqq 1-\frac{\Delta^{\alpha}}{2 A^{\alpha}}, \quad|\xi|>\Delta .
$$

To prove (28), let us assume it is false. Then we can find a sequence $A_{n} \rightarrow \infty$ and for each $n$ a $\xi_{n}$ with $\left|\xi_{n}\right|>\Delta$ such that

$$
\hat{k}\left(\frac{\xi_{n}}{A_{n}}\right)>1-\frac{\Delta^{\alpha}}{2 A_{n}^{\alpha}} .
$$

By choosing a subsequence we may assume that $\xi_{n} / A_{n}$ converges to a limit $l$, which may be $\pm \infty$. If $l=0$ we have for sufficiently large $n$,

$$
\hat{k}\left(\frac{\xi_{n}}{A_{n}}\right) \leqq 1-\frac{1}{2}\left|\frac{\xi_{n}}{A_{n}}\right|^{\alpha}<1-\frac{\Delta^{\alpha}}{2 A_{n}^{\alpha}}
$$


which contradicts (29). If $l \neq 0$ then $\lim \hat{k}\left(\xi_{n} / A_{n}\right)<1$, whereas (29) gives $\lim \hat{k}\left(\xi_{n} / A_{n}\right) \geqq 1$. Thus (29) is false and the estimate (28) is established.

It follows from (27) and (28) that

$$
\begin{aligned}
\mu_{A} & \leqq \int_{-\Delta}^{\Delta}\left|\Psi_{A}(\xi)\right|^{2} d \xi+\left(1-\frac{\Delta^{\alpha}}{2 A^{\alpha}}\right) \int_{|\xi|>\Delta}\left|\Psi_{A}(\xi)\right|^{2} d \xi \\
& =1-\frac{\Delta^{\alpha}}{2 A^{\alpha}}+\frac{\Delta^{\alpha}}{2 A^{\alpha}} \int_{-\Delta}^{\Delta}\left|\Psi_{A}(\xi)\right|^{2} d \xi
\end{aligned}
$$

and so

$$
\int_{-\lrcorner}^{د}\left|\Psi_{A}(\xi)\right|^{2} d \xi \geqq 1-\frac{2 A^{\alpha}}{\Delta^{\alpha}}\left(1-\mu_{A}\right) \geqq 1-\frac{2 \gamma}{\Delta^{\alpha}},
$$

by (26). Now assume that as $A \rightarrow \infty, \psi_{A}$ converges weakly to $\psi$. Then $\Psi_{A}(\xi)$ converges boundedly to $\Psi(\xi)=(2 \pi)^{-1 / 2} \int_{-1}^{1} e^{i \xi x} \psi(x) d x$. Therefore

$$
\int_{-\infty}^{\infty}|\Psi(\xi)|^{2} d \xi \geqq \int_{-\Delta}^{\Delta}|\Psi(\xi)|^{2} d \xi \geqq 1-\frac{2 \gamma}{\Delta^{\alpha}} .
$$

Since $\Delta$ is arbitrarily large we obtain

$$
\int_{-\infty}^{\infty}|\Psi(\xi)|^{2} d \xi \geqq 1
$$

Since the opposite inequality is trivial, we have

$$
\int_{-1}^{1}|\psi(x)|^{2} d x=1
$$

This, in conjunction with weak convergence to $\psi$, gives mean square convergence.

LEMmA 2. $\operatorname{Lim} \sup _{A \rightarrow \infty} \mu_{1, A}^{\left[A^{\alpha}\right]} \leqq e^{-1 / \lambda_{1}}$.

Proof. We may assume $\lim \sup \mu_{1, A}^{\left[A^{\alpha}\right]}=2 L>0$, for otherwise the lemma holds trivially. Choose a sequence of $A$ 's tending to infinity in such a way that $\mu_{1, A}^{\left[A^{\alpha}\right]}$ converges to $\lim$ sup $\mu_{1, A}^{\left[A^{\alpha}\right]}$. Then for sufficiently large $A$ we shall have

$$
\mu_{1, A}>1-A^{-\alpha} \log L .
$$

If now we choose a subsequence such that $\psi_{1, A}$ converges weakly to a function $\psi_{1}$, Lemma 1 tells us that the convergence is in fact strong.

If $P$ is a continuous function vanishing for $u \leqq e^{-1 / \lambda_{1}},(25)$ gives

$$
\lim _{A \rightarrow \infty} \sum_{j=-\infty}^{\infty} P\left(\mu_{j, A}^{\left[A^{\alpha}\right]}\right) \psi_{j, A}(x) \int_{-1}^{y} \psi_{j, A}(x) d x=0 .
$$


Now as $A \rightarrow \infty$ through the sequence chosen above, the strong convergence of $\psi_{1, A}$ implies

$$
\lim _{A \rightarrow \infty} P\left(\mu_{1, A}^{\left[A^{\alpha}\right]}\right) \int_{-1}^{y} \psi_{1, A}(x) d x=0
$$

Since

$$
\lim _{A \rightarrow \infty} \int_{-1}^{y} \psi_{1, A}(x) d x=\int_{-1}^{y} \psi_{1}(x) d x,
$$

which is not identically zero in $y$, we must have

$$
P\left(\lim \sup \mu_{1, A}^{\left[A^{\alpha}\right]}\right)=0 .
$$

This holds for any continuous $P$ vanishing for $u \leqq e^{-1 / \lambda_{1}}$, so the lemma is established.

For the next lemma recall our division of the positive integers into blocks $J_{1}, J_{2}, \cdots$. We denote by $j_{1}$ the largest integer in $J_{1}$. (Thus $j_{1}$ is the multiplicity of $\lambda_{\mathbf{1}}$.)

Lemma 3. Assume that for a sequence of $A$ 's tending to infinity we have

(i) $\mu_{k, A}^{[A \alpha} \rightarrow e^{-1 / \lambda_{1}}$ for $k \leqq k_{0}$,

(ii) $\psi_{k, A}$ converges weakly to $\psi_{k}$ for $k \leqq k_{0}$,

(iii) $\lim \sup \mu_{k_{0}+1, A}^{[\alpha]}<e^{-1 / \lambda_{1}}$.

Then each of $\phi_{1}, \cdots, \phi_{j_{1}}$ is a linear combination of $\psi_{1}, \cdots, \psi_{k_{0}}$.

Proof. Let $P$ be a continuous function equal to 1 for $u=e^{-1 / \lambda_{1}}$ but vanishing for

$$
u \leqq \frac{1}{2}\left(\lim \sup \mu_{k_{0}+1, A}^{\left[A^{\alpha}\right]}+e^{-1 / \lambda_{1}}\right) .
$$

With this $P$ we obtain from (25), after multiplying by $\phi_{j}\left(x_{0}\right)\left(j \leqq j_{1}\right)$ and integrating over $(-1,1)$,

$$
\lim _{A \rightarrow \infty} \sum_{k=1}^{k_{0}} P\left(\begin{array}{c}
{\left[A_{k, A}^{\alpha}\right]} \\
\mu_{k}
\end{array}\right)\left(\psi_{k, A}, \phi_{j}\right) \int_{-1}^{\nu} \psi_{k, A}(x) d x=\int_{-1}^{\nu} \phi_{j}(x) d x .
$$

Therefore

$$
\sum_{k=1}^{k_{0}}\left(\psi_{k}, \phi_{j}\right) \int_{-1}^{\nu} \psi_{k}(x) d x=\int_{-1}^{y} \phi_{j}(x) d x,
$$

and so

$$
\sum_{k=1}^{k_{0}}\left(\psi_{k}, \phi_{j}\right) \psi_{k}(x)=\phi_{j}(x)
$$


Leмma 4. Assume that for a sequence of $A$ 's tending to infinity we have

(i) $\mu_{\mathbf{k}, A}^{[A \alpha]} \rightarrow e^{-1 / \lambda_{1}}$ for $k \leqq k_{0}$,

(ii) $\psi_{k, A}$ converges weakly to $\psi_{k}$ for $k \leqq k_{0}$.

Then each of $\psi_{1}, \cdots, \psi_{k_{0}}$ is a linear combination of $\phi_{1}, \cdots, \phi_{j_{1}}$.

Proof. Since by Lemma $1 \psi_{k, A}$ converges in mean square to $\psi_{k}$ we obtain from (25)

$$
\lim _{A \rightarrow \infty} F\left(\mu_{k, A}^{\left[A^{\alpha}\right]}\right) \int_{-1}^{y} \psi_{k, A}(x) d x=\sum_{j=1}^{\infty} F e{\overline{\left(1 / \lambda_{j}\right.}}^{-1}\left(\psi_{k}, \phi_{j}\right) \int_{-1}^{y} \phi_{j}(x) d x .
$$

Choose $F$ so that $F\left(e^{-1 / \lambda_{1}}\right)=1$ but $F(u)=0$ for $u \leqq e^{-1 / \lambda_{i_{2}+1}}$. Then we obtain

$$
\int_{-1}^{y} \psi_{k}(x) d x=\sum_{j=1}^{j_{1}}\left(\psi_{k}, \phi_{j}\right) \int_{-1}^{y} \phi_{j}(x) d x,
$$

and so

$$
\psi_{k}(x)=\sum_{j=1}^{i_{1}}\left(\psi_{k}, \phi_{j}\right) \phi_{j}(x) .
$$

Now we can show that

$$
\lim _{A \rightarrow \infty} \mu_{k, A}^{\left[A^{\alpha}\right]}=e^{-1 / \lambda_{1}}
$$

$k \leqq j_{1}$

Let $k_{0}+1\left(k_{0} \geqq 0\right)$ be the smallest integer $k$ for which the identity fails to hold. Then, in view of Lemma 2 , we can find a sequence of $A$ 's tending to infinity so that the limit of $\mu_{k_{0}+1, A}^{\left[x^{\gamma}\right]}$ exists and is less than $e^{-1 / \lambda_{1}}$. But we have

$$
\lim \mu_{k, A}^{\left[A^{\alpha}\right]}=e^{-1 / \lambda_{1}}
$$$$
k \leqq k_{0} \text {. }
$$

Therefore by Lemma 3 we have $k_{0} \geqq j_{1}$.

We have also shown (see (30), (31), and Lemma 1) that any sequence of $A$ 's tending to infinity possesses a subsequence for which

$$
\begin{array}{ll}
\phi_{j}(x)-\sum_{k=1}^{i_{1}}\left(\psi_{k, A}, \phi_{j}\right) \psi_{k, A}(x) \rightarrow 0, & \left(j \leqq j_{1}\right) \\
\psi_{k, A}(x)-\sum_{j=1}^{j_{1}}\left(\psi_{k, A}, \phi_{j}\right) \phi_{j}(x) \rightarrow 0, & \left(k \leqq j_{1}\right)
\end{array}
$$

in mean square. It follows that (32) holds as $A$ tends continuously to infinity, and this proves the convergence of the space $\mathfrak{N}_{1, A}$ to $\mathfrak{T}_{1}$.

Using (25) and what has already been proved, we obtain

$$
\lim _{A \rightarrow \infty} \sum_{j \leq 0 \text { or }>j_{1}} P\left(\mu_{j, A}^{\left[A^{\alpha}\right]}\right) \psi_{j, A}\left(x_{0}\right) \int_{-1}^{\nu} \psi_{j, A}(x) d x=\sum_{j>j_{1}} P\left(e^{-1 / \lambda}\right) \phi_{j}\left(x_{0}\right) \int_{-1}^{y} \phi_{j}(x) d x .
$$


Thus we can repeat the argument and show that

$$
\lim _{A \rightarrow \infty} \mu_{j, A}^{\left[A^{\alpha}\right]}=e^{-1 / \lambda j_{1}+1}, \quad j_{1}<j \leqq j_{2},
$$

where $j_{2}$ is the largest integer in $J_{2}$, and that $\mathfrak{N}_{2, A}$ converges to $\mathfrak{T}_{2}$. The complete result clearly follows by induction.

3.5. It is easy to conjecture what happens when $\alpha>2$ in (11). Now $k$ is not positive, but a reasonable set of hypotheses is: $k$ is real, even, and integrable. Moreover

$$
\begin{aligned}
& \max \hat{k}(\xi)=\hat{k}(0)=1, \quad \hat{k}(\xi)<1 \text { for } \xi \neq 0, \\
& \lim _{\xi \rightarrow 0} \frac{1-\hat{k}(\xi)}{|\xi|^{\alpha}}=c, \quad(0<c<\infty) .
\end{aligned}
$$

Then the theorem stated in $\$ 3.1$ ought to hold, with a suitable definition of $K(x, y)$ for $\alpha>2$. Now it is easily seen that for fixed $x, y, K(x, y)$ car be extended to be analytic in the half-plane $R \alpha>0$, and it is this extension which should serve the purpose.

There is independent support for this conjecture. A straightforward computation shows that for $\alpha=4$ (when $K$ is the Green's function for the operator $u \rightarrow u^{\text {(iv) }}$ with boundary conditions $u(-1)-u^{\prime}(-1)=u(1)=u^{\prime}(1)$ $=0)$ the eigenvalues are the reciprocals of the fourth powers of the roots of

$$
\tan ^{2} r_{j}=\tanh ^{2} r_{j}
$$

Thus the conjecture concerning the eigenvalues in case $\alpha=4$ is

$$
\mu_{j, A}=1-\operatorname{cr}_{j}^{4} A^{-4}+o\left(A^{-4}\right) .
$$

The discrete version of this, a limit theorem for the eigenvalues of Toeplitz matrices, was in fact proved by Parter [9].

Added in proof: The general conjecture is proved in a forthcoming paper.

\section{REFERENCES}

1. T. Carleman, Über die Abelsche Integralgleichung mit konstanten Integrationsgrenzen, Math. Z. vol. 15 (1922) pp. 111-120.

2. J. Elliott, Absorbing barrier processes connected with the symmetric stable densities, Illinois J. Math. vol. 3 (1959) pp. 200-216.

3. P. Erdös and M. Kac, On certain limit theorems of the theory of probability, Bull. Amer. Math. Soc. vol. 52 (1946) pp. 292-302.

4. R. K. Getoor, First passage times for symmetric stable processes in space, Trans. Amer. Math. Soc., to appear.

5. M. Kac, On some connections between probability theory and differential and integral equations, Proceedings of the Second Berkeley Symposium on Mathematical Statistics and Probability, Berkeley and Los Angeles, University of California Press, 1951, pp. 189-215.

6. M. Kac, W. L. Murdock and G. Szegö, On the eigenvalues of certain Hermitian forms, J. Rational Mech. Anal. vol. 2 (1953) pp. 767-800. 
7. M. Kac and H. Pollard, The distribution of the maximum of partial sums of independent random variables, Canad. J. Math. vol. 2 (1950) pp. 375-384.

8. E. G. Kimme, On the convergence of sequences of stochastic processes, Trans. Amer. Math. Soc. vol. 84 (1957) pp. 208-229.

9. S. V. Parter, Extreme eigenvalues of Toeplitz forms and applications to elliptic difference equations, Trans. Amer. Math. Soc., to appear.

10. D. Ray, Stable processes with an absorbing barrier, Trans. Amer. Math. Soc. vol. 89 (1958) pp. 16-24.

11. F. Spitzer, Some theorems concerning 2-dimensional Brownian motion, Trans. Amer. Math. Soc. vol. 87 (1958) pp. 187-197.

12. H. Widom, On the eigenvalues of certain Hermitian operators, Trans. Amer. Math. Soc. vol. 88 (1958) pp. 491-522.

Cornell University, ITHACA, NEW YORK 